\title{
Silicon strained layers grown on GaP(001) by molecular beam epitaxy
}

Cite as: Journal of Applied Physics 58, 3097 (1985); https://doi.org/10.1063/1.335811

Submitted: 03 June 1985 . Accepted: 14 June 1985 . Published Online: 04 June 1998

P. M. J. Marée, R. I. J. Olthof, J. W. M. Frenken, J. F. van der Veen, C. W. T. Bulle-Lieuwma, M. P. A. Viegers, and P. C. Zalm

ARTICLES YOU MAY BE INTERESTED IN

Incoherent interface of InAs grown directly on GaP(001)

Applied Physics Letters 69, 981 (1996); https://doi.org/10.1063/1.117102

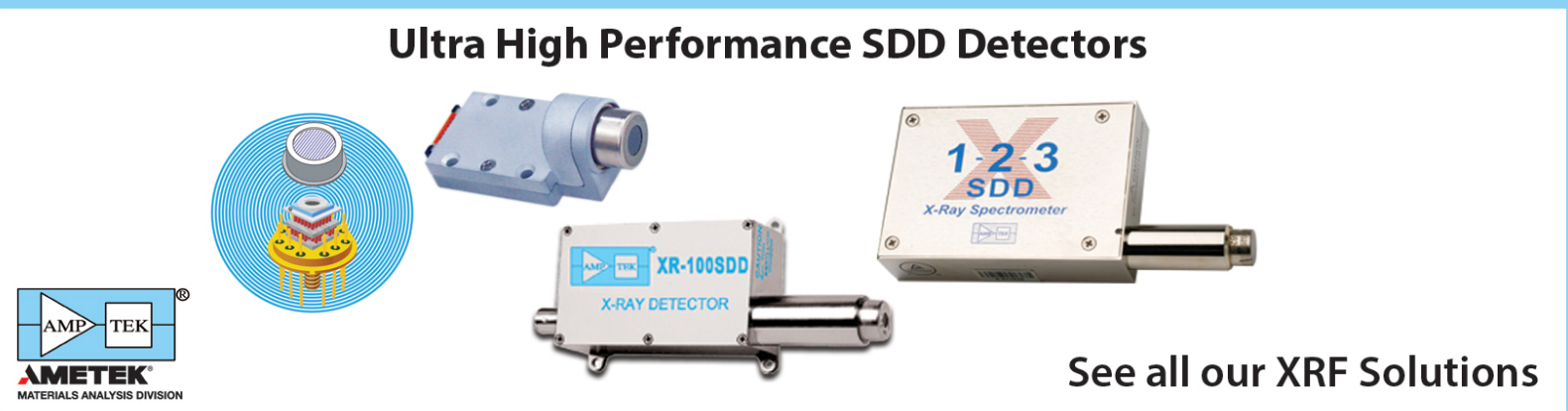




\title{
Silicon strained layers grown on GaP(001) by molecular beam eplaxy
}

\author{
P. M. J. Maree, R. I. J. Olthof, J. W. M. Frenken, and J. F. van der Veen \\ F.O.M. Institute for Atomic and Molecular Physics, Kruislaan 407, 1098 SJ Amsterdam, The Netherlands
}

C. W. T. Bulle-Lieuwma, M. P. A. Viegers, and P. C. Zalm

Philips Research Laboratories, P. O. Box 80000,5600 JA Eindhoven, The Netherlands

(Received 3 June 1985; accepted for publication 14 June 1985)

Mismatch-induced lattice strain in thin Si films grown by molecular beam epitaxy on $\mathrm{GaP}(001)$ substrates has been measured using transmission electron microscopy, Raman spectroscopy, and Rutherford backscattering. The perpendicular strain in the topmost part of the layers is found to be enhanced in comparison to elasticity theory. Relaxation of the strain occurs by the formation of misfit dislocations at significantly larger thickness than predicted by equilibrium theory.

\section{INTRODUCTION}

Strained-layer heteroepitaxy offers possibilities to semiconductor technology for the realization of advanced devices. Heterostructures, in which materials with different band gaps are combined in a single device, allow for independent control of electrons and holes. ${ }^{1}$ Moreover, the difference in lattice constants of the semiconductor materials leads to strain in the overlayer, which can change the electrical and optical properties, ${ }^{2}$ such as resistivity, band gap, Hall mobility, and refractory index. This capability of modifying material properties offers even more freedom in the design of semiconductor devices. Use has been made of these principles in, for instance, semiconductor strained-layer superlattices. $^{3-6}$ These are multilayered structures of semiconductors with a slight lattice mismatch.

The lattice strain is inherent to lattice-mismatched heterostructures. Although useful with respect to the modification of electrical and optical properties, it can severely affect the material structure, leading to unwanted crystalline defects. ${ }^{6}$ In particular it will influence the growth of layers of large mismatch and/or large thickness. ${ }^{7}$ Even if initially a pseudomorphic layer can be formed, when growth is continued, the strain will eventually be released through the formation of misfit dislocations, which will influence the electrical behavior of semiconductors in several ways. (They can provide short circuit diffusion paths or preferred sites for dopants, or act as recombination centers for charge carriers.) An extensive study of the phenomenon of strain release by misfit dislocations has been performed for the $\mathrm{Ge}_{x} \mathrm{Si}_{1-x}: \mathrm{Si}(001)$ system. ${ }^{8-12}$

The need to measure strain arises from several reasons. Strained layers are interesting materials as such. Their properties are dependent on the amount of strain, so knowledge of this quantity is important. Experimental data are necessary because elasticity theory might deviate for extremely thin layers. Furthermore, the strain is related to the formation of misfit dislocations.

We have measured the tensile strain in layers of elemental Si of various thicknesses grown on $\mathrm{GaP}(001)$ substrates by molecular beam epitaxy (MBE). The lattice mismatch of this system is $3.6 \times 10^{-3}$. The resulting strain could give rise to an enhancement of the mobility in the silicon film, which we will discuss later. Strain is measured by means of Raman spectroscopy and Rutherford backscattering (RBS), dislocation densities are obtained by transmission electron microscopy (TEM). The results are compared with predictions of the equilibrium theory of dislocation generation.

\section{SAMPLE PREPARATION}

For the fabrication of the heteroepitaxial Si layers on $\mathrm{GaP}$ a low-temperature technique such as $\mathrm{MBE}$ is needed in order to obtain an abrupt interface and to prevent decomposition of the GaP substrate. It has been shown by De Jong et $a l .{ }^{13}$ that crystalline Si layers of good quality can be grown on $\mathrm{GaP}(001)$.

The GaP samples were ultrasonically rinsed in highpurity ethanol and bonded with small amounts of indium to a Si carrier sample, which could be resistively heated. The samples were introduced in a Si-MBE apparatus described elsewhere. ${ }^{14}$ In the ultrahigh vacuum (UHV) system the GaP substrates were inspected by means of Auger electron spectroscopy (AES), showing $\mathrm{Ga}(55 \mathrm{eV})$ and $P(120 \mathrm{eV})$ as well as some $C(270 \mathrm{eV})$ and $\mathrm{O}(509 \mathrm{eV})$. No In was observed at the front side of the sample in any stage of the experiment. The cleaning procedure consisted of sputtering with $0.8-\mathrm{keV}$ $\mathrm{Ar}^{+}$ions at an angle of $60^{\circ}$ with the surface plane to a total dose of about $2 \times 10^{16} \mathrm{~cm}^{-2}$, and subsequent thermal annealing for $30 \mathrm{~min}$ at $570^{\circ} \mathrm{C}$. After this treatment neither $\mathrm{C}$ nor $O$ could be detected by AES (detection limits 1 and $2 \%$ of a monolayer, respectively) and the low-energy electron diffraction (LEED) pattern showed the familiar Ga stabilized $c(8 \times 2)$ reconstruction. On these $G a P(001)$ substrates Si was deposited at $570^{\circ} \mathrm{C}$ at a rate of approximately $1 \AA / \mathrm{s}$ to thicknesses up to $4000 \AA$. After growth a sharp $2 \times 1$ LEED pattern was observed. The AES signal showed small amounts ( $\leqslant 1$ monolayer) of segregated $\mathrm{Ga}$ and $\mathrm{P}$ on top of the Si layers. For further characterization the samples were removed from the UHV system.

RBS with $2-\mathrm{MeV} \mathrm{He}^{+}$ions showed a channeling minimum yield of $3 \%$, which indicates good crystalline order. This is confirmed by TEM micrographs which show the perfect extension of the lattice planes of the substrate into the epitaxial layers (Fig. 1), and a very low density $\left(<10^{4} \mathrm{~cm}^{-2}\right)$ of dislocations threading through the Si layer. 


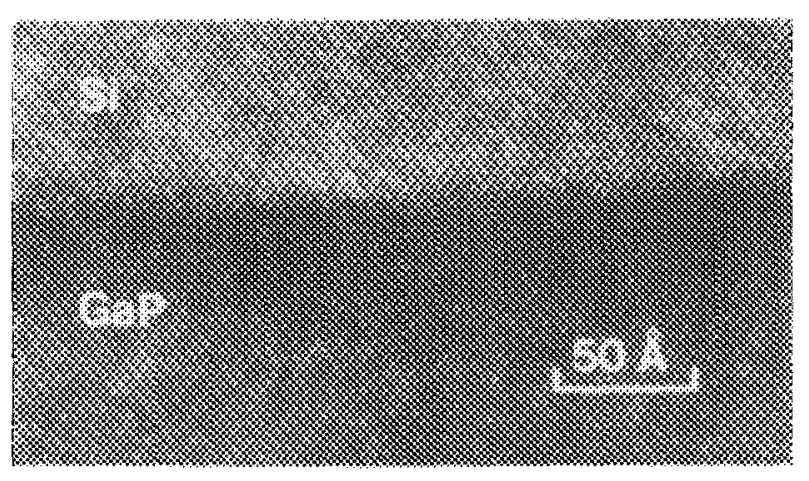

FIG. 1. High-resolution TEM image of the $\mathrm{Si}: \mathrm{GaP}(001)$ interfacial region, viewed in a $\langle 011\rangle$ cross section.

\section{IIII. CONSEQUENCES OF THE LATTICE MISMATCH}

The lattice mismatch between epitaxial film and substrate is defined as

$$
f=\left(a_{s}-a_{f}\right) / a_{f},
$$

where $a_{s}$ and $a_{f}$ are the lattice constants of substrate and film, respectively. For the Si-GaP system, the misfit at room temperature is equal to $f_{R T}=3.6 \times 10^{-3}$ and at the growth temperature $f_{570^{\circ} \mathrm{C}}=4.6 \times 10^{-3}$, because of the difference in linear expansion coefficients. ${ }^{15}$ The lattice mismatch is shared between elastical strain parallel to the growth plane $\left(\epsilon_{\|}\right)$and misfit dislocations. Assuming a low density and an isotropic and homogeneous distribution of misfit dislocations this implies:

$$
f=\epsilon_{\|}+\delta \text {. }
$$

The part of the lattice mismatch that is accommodated by misfit dislocations $(\delta)$ is related to their mean-separation distance $(d)$ and the edge component of the Burgers vector projected at the interface $\left(b_{\|}\right)$by

$$
\delta=b_{\|} / d .
$$

If the misfit is completely relaxed by misfit dislocations their mean-separation distance would be $d=b_{\|} / f$, which for the $\mathrm{Si}: \mathrm{GaP}(001)$ system is equal to $d=520 \AA$ at room temperature.

For thin films-and in case the lattice mismatch is not too large-growth is pseudomorphic or coherent, i.e., the layer is strained elastically in such a way that the lattices of film and substrate are in register at the interface.

Above a certain thickness $t_{c}$ part of the misfit is accommodated by misfit dislocations. This process has been studied experimentally among others for the $\mathrm{Si}_{x} \mathrm{Ge}_{1-x}: \mathrm{Si}(001)$ system $^{8-12}$ and is theoretically described in the equilibrium theory ${ }^{7.9,16,17}$ of Van der Merwe. This equilibrium theory is based on energy considerations only. The energy of homogeneous strain per unit interfacial area is ${ }^{16,17}$

$$
E_{\epsilon_{\|}}=\left[2 \mu_{f}(1+v) /(1-v)\right] \epsilon_{\|}^{2} t
$$

and the energy of a square grid of perpendicular dislocation lines per unit area is approximately: ${ }^{17}$

$$
E_{\delta}=\frac{\mu_{f} \mu_{s}}{\pi\left(\mu_{f}+\mu_{s}\right)(1-v)} b_{\|}\left(f-\epsilon_{\|}\right)\left(1+\ln \frac{R}{b_{\|}}\right),
$$

where $\mu_{f}$ and $\mu_{s}$ are the shear moduli of film and substrate, $v$ Poisson's ratio of the film, $t$ the layer thickness, and $R$ the effective range of the field of a misfit dislocation. For lowdislocation densities $R=t$. When the number of dislocations has increased to the point that the conditions $d \leqslant 2 t$ is fulfilled, then their fields are screened by those of neighboring dislocations and the range becomes $R=\frac{1}{2} d=b_{\|} / 2\left(f-\epsilon_{\|}\right)$. The value of $\epsilon_{\|}$for which the sum of $E_{\epsilon_{\|}}$and $E_{\delta}$ is minimum is ${ }^{17}$

$$
\epsilon_{\|}^{0}=\frac{\mu_{s} b_{\|}}{4 \pi\left(\mu_{f}+\mu_{s}\right)(1+v) t} A,
$$

where the function

$$
A=\left[\begin{array}{cc}
1+\ln \left(t / b_{\|}\right), & \text {for } d>2 t, \\
-\ln 2\left(f-\epsilon_{\|}^{0}\right), & \text { for } d \leqslant 2 t .
\end{array}\right.
$$

The elaborate expression for $E_{\delta}$ derived by Van der Merwe ${ }^{16}$ is more accurate than $\mathrm{Eq}$. (5) only in the high-dislocation density regime. If this expression is used $A$ is a complicated function of $f, \epsilon_{\| l}$, and the elastic constants. In that case, even lower values of $\epsilon_{\|}^{0}$ are found ${ }^{17}$ than would follow from Eq. (6). According to the equilibrium theory the elastic strain in a film of certain thickness $t$ is described by $\epsilon_{\|}=\epsilon_{\|}^{0}$, insofar as $\epsilon_{\|}$does not exceed $f$, in which case $\epsilon_{\|}=f$. The calculated thickness dependence of $\epsilon_{\|}$for Si on GaP(001) is shown in Fig. 9. The thickness $t_{c}$ at which the first misfit dislocations are generated, is called critical thickness. At $t=t_{c}$ [calculated from Eq. (6) by putting $\left.\epsilon_{\|}^{0}=f\right\rfloor \epsilon_{\|}$begins to deviate from $f$. For Si on GaP the equilibrium theory predicts $t_{c}=140$ $\AA$.

\section{ELASTICITY}

Thus far we have only discussed the parallel component of the strain in the layer $\epsilon_{\|}$, imposed on the epitaxial film by the lattice mismatch and the degree of registry between film and substrate at the interface. We now want to take into account the other strain components as well. We therefore consider the relations among them, such as given by elasticity theory. The generalized Hooke's law relates the stress tensor $S$ with the strain tensor $\epsilon$ :

$$
\mathrm{S}=\overline{\mathrm{C}}: \epsilon \text {. }
$$

They are coupled by the fourth rank tensor $\bar{C}$. The $81 \mathrm{com}-$ ponents of $\bar{C}$, the elastic constants, are strongly reduced in number by symmetry to only three independent components for a cubic crystal. The same reduction appears for the diamond crystal structure if the stress is applied in a $\{100\}$ plane. ${ }^{18}$ We choose a coordinate system with $x$ and $y$ axes in the film plane and $z$ axis in the growth direction. The elasticity relations are now given by the following set of equations:

$S_{x x}=C_{11} \epsilon_{\|}+C_{12}\left(\epsilon_{\|}+\epsilon_{1}\right)$,

$S_{y y}=C_{11} \epsilon_{\|}+C_{12}\left(\epsilon_{\|}+\epsilon_{1}\right)$,

$S_{z z}=C_{11} \epsilon_{\perp}+2 C_{12} \epsilon_{\|}$,

$S_{x y}=C_{44} \epsilon_{x y}, \quad S_{y z}=C_{44} \epsilon_{y z}, \quad S_{z x}=C_{44} \epsilon_{z x}$.

In the case of a biaxial strain field $S_{x x}=S_{y y}$ and $S_{z z}=S_{x y}=S_{y z}=S_{z x}=0$, which leads to:

$$
\epsilon_{\perp}=-\alpha \epsilon_{\|} \text {with } \alpha=2\left(C_{12} / C_{11}\right) \text {. }
$$


This is the Poisson effect, which describes the contraction of the layer in the direction normal to the applied tetragonal stress. Numerical values of the $\mathrm{Si}$ bulk elastic constants ${ }^{18}$ are $C_{11}=16.58 \times 10^{10} \mathrm{~Pa}$ and $C_{12}=6.39 \times 10^{10} \mathrm{~Pa}$. Thus, if elasticity theory applies we would have $\alpha=0.77$. If instead we had conservation of volume this would imply

$$
1+\epsilon_{1}=1 /\left(1+\epsilon_{\|}\right)^{2} \approx 1-2 \epsilon_{\|},
$$

giving $\alpha=2$. If, on other hand, we required the bond lengths, all pointing in $\langle 111\rangle$ directions, to be rigid, this would give

$$
1+\epsilon_{1}=\sqrt{3-2\left(1+\epsilon_{\|}\right)^{2}} \approx 1-2 \epsilon_{\|},
$$

which would also yield $\alpha=2$.

\section{STRAIN MEASUREMENTS}

TEM, Raman, and RBS were applied to measure the strain in the Si overlayer. Each of these techniques is sensitive to a certain component or to a combination of components of the strain tensor.

\section{A. Transmission electron microscopy and $x$-ray diffraction}

TEM observations in the planar mode have shown that layers with thicknesses up to $500 \AA$ are completely free of misfit dislocations (detection limit $\approx 10^{4} \mathrm{~cm}^{-2}$ ) indicating a pseudomorphic state of Si on $\mathrm{GaP}(001)$. For a 2000 - $\AA$-thick layer a square grid of dislocation lines on the [110] and [110] directions is seen (Fig. 2). It concerns $60^{\circ}$ dislocations, lying on $\{111\}$ planes, with Burgers vectors of the type $\frac{1}{2} a_{f}\langle 110\rangle$ inclined with respect to the interface plane. The out-of-plane components, however, average out, so that the mean displacement associated with each dislocation is given by $b_{\|}=\frac{1}{4} a_{f} \sqrt{2}=1.94 \AA$. Measured values for the mean-separation distance $\bar{d}$ are given in Table I. $\delta$ and $\epsilon_{\|}$can be calculated from $\bar{d}$ and the known values of $f$ and $b_{\text {lacording }}$ to Eqs. (2) and (3) (results are given in Fig. 9, in which the thickness dependence of $\epsilon_{\text {as measured by various techniques is }}$ compared with equilibrium theory calculations).

With double-crystal $x$-ray diffractometry using the $\mathrm{Cu}$ $\mathrm{X}_{\alpha_{1}}$ (004) reflection, it was established that the average lattice constant normal to the interface of a $2-\mu \mathrm{m}$-thick Si film

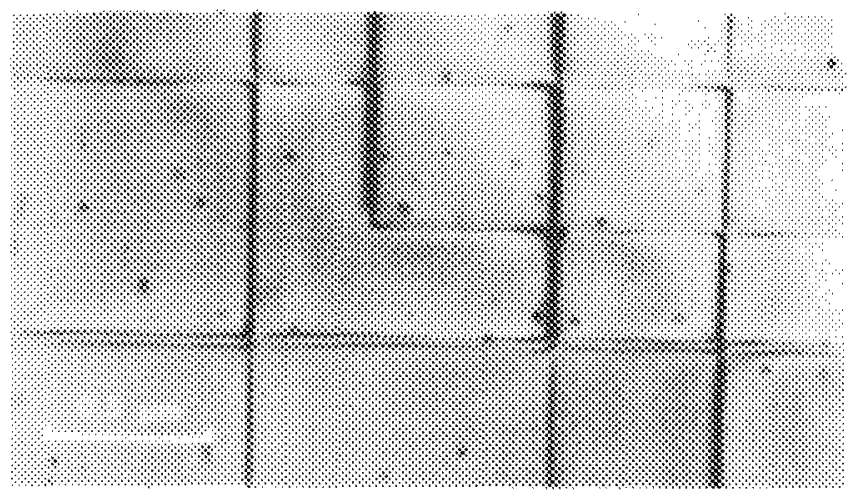

FIG. 2. Transmission electron micrographs of a 2000-Å Si film on GaP, viewed in the [00 $\overline{1}]$ direction. Dislocation lines run along the interface in (110) directions.
TABLE I. Mean separation distance $\bar{d}$ between misfit dislocations obtained

\begin{tabular}{|c|c|c|}
\hline $\begin{array}{c}t \\
{[\AA ̊}\end{array}$ & $\left.\begin{array}{c}\bar{d} \\
{[\AA]}\end{array}\right]$ & $\begin{array}{c}\delta \\
{\left[\times 10^{-3}\right]}\end{array}$ \\
\hline 500 & $\infty$ & 0 \\
\hline 2000 & 3400 & 0.55 \\
\hline
\end{tabular}
with TEM.

is completely relaxed to the unstrained (bulk) value (Fig. 3). This confirmed that the average strain is zero in these thicker layers:

$$
\epsilon_{\perp}=0 \text { for } t>>t_{c} .
$$

We ascribe the broadening of the peak to a high density of dislocations and their localized inhomogeneous strain fields.

\section{B. Raman spectroscopy}

Raman scattering experiments were performed in the reflection geometry using the 5145 - $\AA$ line of a $\mathrm{Ar}^{+}$ion laser. The Raman line of the zone-center $(k \approx 0)$ optical phonon in Si layers of varying thickness (see Fig. 4) was measured at room temperature with a spectral resolution of $1.6 \mathrm{~cm}^{-1}$ full width at half-maximum (FWHM). The results of the Raman spectroscopy measurements are given in Table II (uncorrected for the instrumental resolution).

The determination of $\omega_{0}$, the Si optical phonon frequency for zero stress, deserves some consideration. The Raman

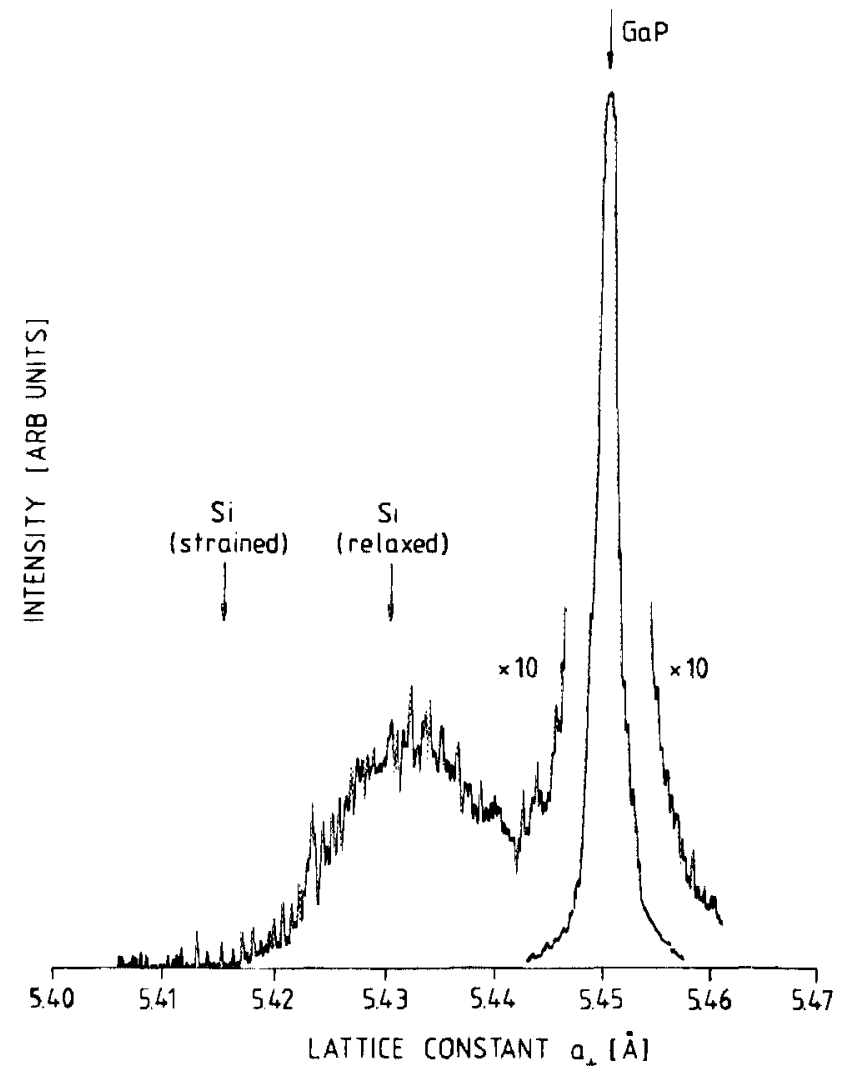

FIG. 3. X-ray rocking curves of the $\mathrm{CuK}_{\alpha_{1}}$ (004) reflection indicating the absence of (perpendicular) strain in a $2-\mu \mathrm{m}$-thick Si layer on $\mathrm{GaP}$. The expected position of the Si peak for a pseudomorphic strained layer according to the Poisson effect is indicated by an arrow. 


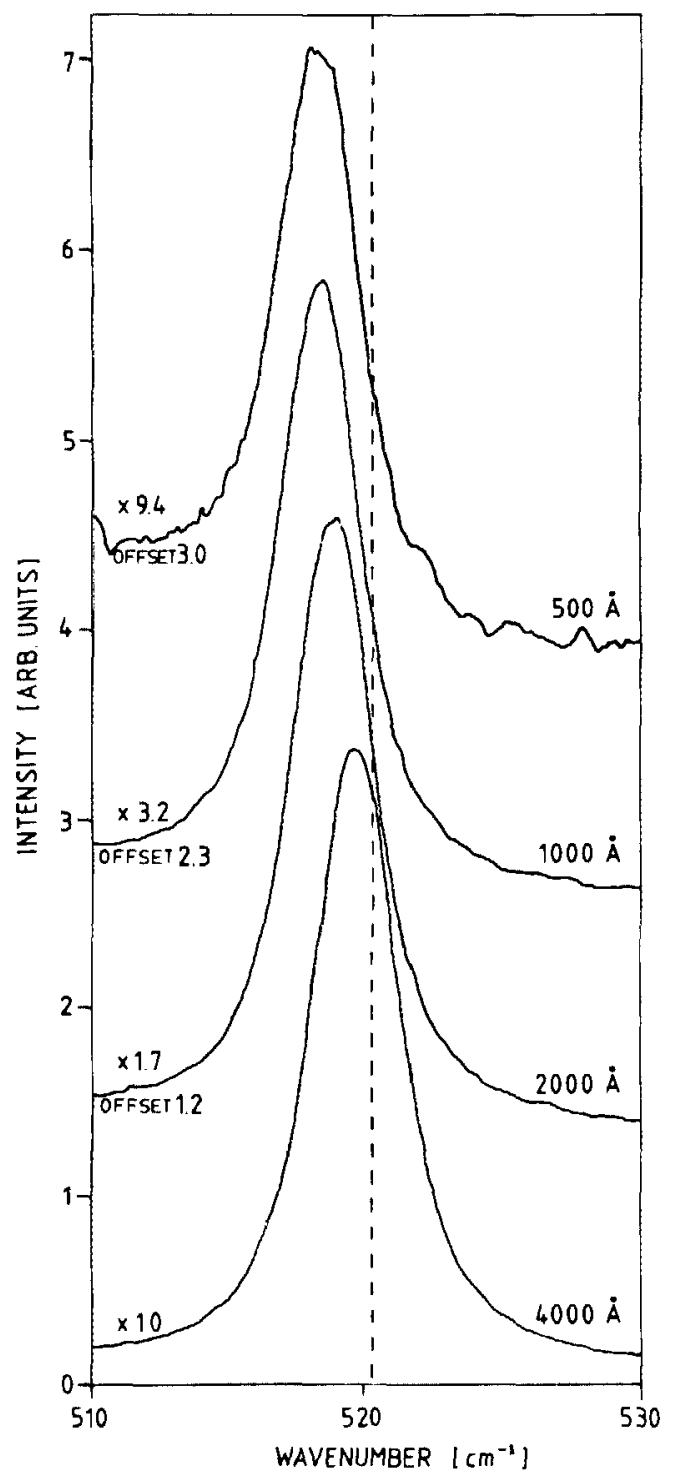

FIG. 4. Raman lines of the Si zone-center $(k \approx 0)$ optical phonon showing the strain-induced frequency shift. The dashed line indicates the value of $\omega$ for bulk $\mathrm{Si}$.

line in bulk Si was initially detected at $520.8 \mathrm{~cm}^{-1}$, clearly because of a temperature effect. ${ }^{19}$ Because the optical-absorption coefficient for the used laser light in $\mathrm{GaP}$ is about an order of magnitude smaller than in $\mathrm{Si}^{20}$ this temperature effect will not have played a role in the thin Si films. This assumption was confermed by the absence of drift of the Raman lines measured on the films. Consequently, we take the phonon frequency in an (hypothetical) unstrained thin $\mathrm{Si}$ film on $\mathrm{GaP}$ to be $520.8 \mathrm{~cm}^{-1}$, resulting in the values for $\delta \omega$ as given in Table II.

The line widths give qualitative indication of possible disorder, dislocation densities, and inhomogeneous strain in the epilayers. ${ }^{21}$ The frequency shift $\delta \omega$ of the Si $(k \approx 0)$ optical phonon is a direct measure for the tetragonal stress in the films and for the strain, since they are related through the elastic constants [Eq. (8)]. For the observed singlet phonon (perpendicular to the surface) the resulting shift is ${ }^{21,22}$

$$
\delta \omega=\left(p / 2 \omega_{0}\right) \epsilon_{\perp}+\left(q / \omega_{0}\right) \epsilon_{\|},
$$

TABLE II. Raman measurements of the Si zone-center optical phonon frequency.

\begin{tabular}{rlcc}
\hline \hline $\left.\begin{array}{c}t \\
\AA \AA\end{array}\right]$ & \multicolumn{1}{c}{$\begin{array}{c}\delta \omega \\
{\left[\mathrm{cm}^{-1}\right]}\end{array}$} & $\begin{array}{c}\text { line width (FWHM) } \\
{\left[\mathrm{cm}^{-1}\right]}\end{array}$ & $\begin{array}{c}\epsilon_{\|} \\
{\left[\times 10^{-3}\right]}\end{array}$ \\
\hline 500 & $-2.60( \pm 0.05)$ & $3.30( \pm 0.05)$ & 3.6 \\
1000 & $-2.45( \pm 0.05)$ & $3.38( \pm 0.05)$ & 3.4 \\
2000 & $-1.9( \pm 0.1)$ & $3.55( \pm 0.1)$ & 2.6 \\
4000 & $-1.2( \pm 0.1)$ & $3.70( \pm 0.1)$ & 1.7 \\
\hline
\end{tabular}

where $\omega_{0}$ is the phonon frequency in unstrained Si. The phenomenological constants $p$ and $q$ are known from Raman scattering experiments under uniaxial stress or hydrostatic pressure ${ }^{22}: p=-1.2 \times 10^{28} \mathrm{~s}^{-2}$ and $q=-1.8 \times 10^{28} \mathrm{~s}^{-2}$, both within $20 \%$. Using Eqs. (10) and (14) the following expression can be derived for the parallel strain component:

$$
\epsilon_{\|}=\beta \delta \omega \quad \text { with } \beta=2 \omega_{0} /(-\alpha p+2 q) .
$$

Taking $\alpha=0.77$ as predicted by elasticity theory the proportionality constant becomes $\beta=-1.4( \pm 0.3) \times 10^{-3} \mathrm{~cm}$. The resulting values for $\epsilon_{\|}$are given in Table II and Fig. 9. The error bars in Fig. 9 are based on the relative uncertainties in the $\delta \omega$ values.

\section{c. kon blockling}

A direct consequence of the tetragonal strain in the epitaxial layer is a tilt $\Delta \theta$ of the non-normal crystallographic axes. ${ }^{12,23.24}$ On simple geometrical grounds we can see that

$\Delta \theta=\left(\epsilon_{\mathbb{1}}-\epsilon_{i}\right) \sin \theta \cos \theta=(1+\alpha) \epsilon_{\mathrm{l}} \sin \theta \cos \theta$,

where $\theta$ is the angle between the [001] surface normal and a certain crystallographic direction. This relation is visualized in Fig. 5 for the [011] direction.

The results of the ion-blocking measurements of the angular shift $\Delta \theta$ of the $\langle 011\rangle$ axis are given in Table III. When $\alpha$ is known (see Sec. VI) values for $\epsilon_{\|}$follow from Eq. (16) (Fig. 9).

The angular shift $\Delta \theta$ is induced by coherency strain as well as by the normal contraction due to the Poisson effect (Fig. 5), as described by Eq. (16).

We have used Rutherford backscattering in conjunction

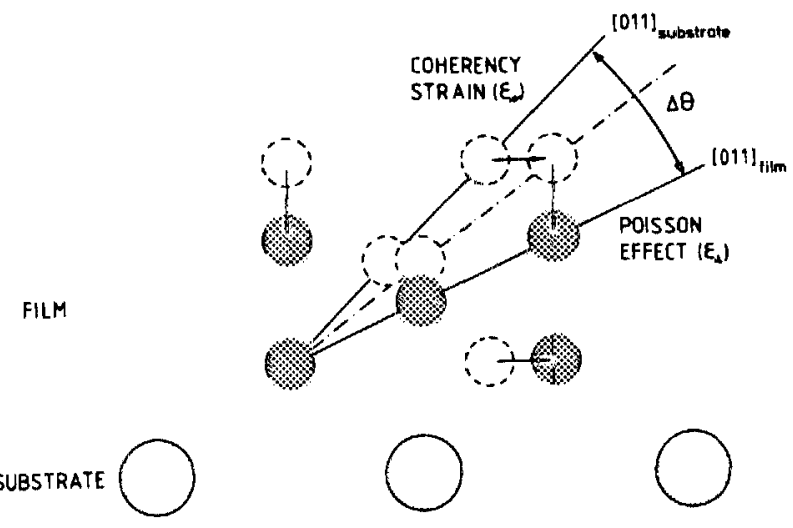

FIG. S. Schematic view of the relation of the angular shift $\Delta \theta$ with both coherency strain $\left(\epsilon_{\|}\right)$and the perpendicular strain $\left(\epsilon_{1}\right)$ due to the Poisson effect. 
TABLE III. Ion-blocking measurements of the $\langle 011\rangle$ angular shift.

\begin{tabular}{|c|c|}
\hline$\stackrel{t}{t}$ & $\begin{array}{c}\Delta \theta \\
\text { [degrees] }\end{array}$ \\
\hline 250 & $0.25( \pm 0.04)$ \\
\hline 300 & $0.26( \pm 0.04)$ \\
\hline 1000 & $0.27( \pm 0.04)$ \\
\hline 2000 & $0.18( \pm 0.04)$ \\
\hline 4000 & $0.14( \pm 0.04)$ \\
\hline$\infty$ & $0.00( \pm 0.04)$ \\
\hline
\end{tabular}

with ion blocking ${ }^{25}$ to measure this angular shift (Fig. 6). The (100) plane was chosen as scattering plane, for it contains both the [001] surface normal, which is unaffected by the strain and will be used as a reference, and the [011] direction, for which the expected shift is largest [Eq. (16)]. In this plane a beam of $\mathrm{H}^{+}$ions with a primary energy of $175 \mathrm{keV}$ was directed onto the sample. An angle of incidence of $10.4^{\circ}$ with respect to the surface plane was chosen in order to avoid axial channeling.

Backscattered protons were analyzed by a toroidal electrostatic analyzer ${ }^{26}$ with an energy resolution of $\Delta E /$ $E=4 \times 10^{-3}$. For $175-\mathrm{keV} \mathrm{H}^{+}$ions in the scattering geometry used this gives a depth resolution of $10 \AA .{ }^{27}$ Energyanalyzed protons were collected by a position-sensitive channelplate detector, enabling simultaneous detection over an angular range of $6^{\circ}$.

In the recorded spectra (Fig. 7) the energy scale was converted to an approximate depth scale for the Si layer by using the random value for the stopping power. ${ }^{27}$ In this procedure the angular dependence of the energy transfer during the collision ${ }^{28}$ has been corrected. For every energy spectrum the yield was normalized by dividing it by the Rutherford cross section. ${ }^{28} \mathrm{~A}$ depth window was set in such a way that only protons scattered from $\mathrm{Si}$ atoms at a depth between 30 and $110 \AA$ contributed (shaded band in Fig. 7). In this way the signal is not disturbed by protons scattered from $\mathrm{Ga}$ or $\mathrm{P}$ atoms in the substrate, from displaced $\mathrm{Si}$ atoms in the thin oxide layer on top of the film, or by protons that have changed their initial direction after the collision with a deeper $\mathrm{Si}$ atom by interaction with $\mathrm{Si}$ atoms from other strings.

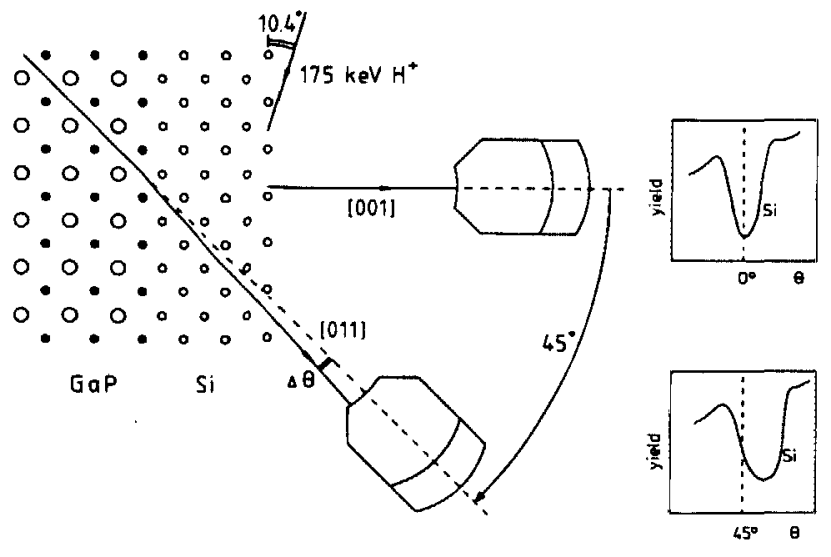

FIG. 6. Schematic view of the ion-blocking experiment, showing the scattering geometry and the measuring procedure.

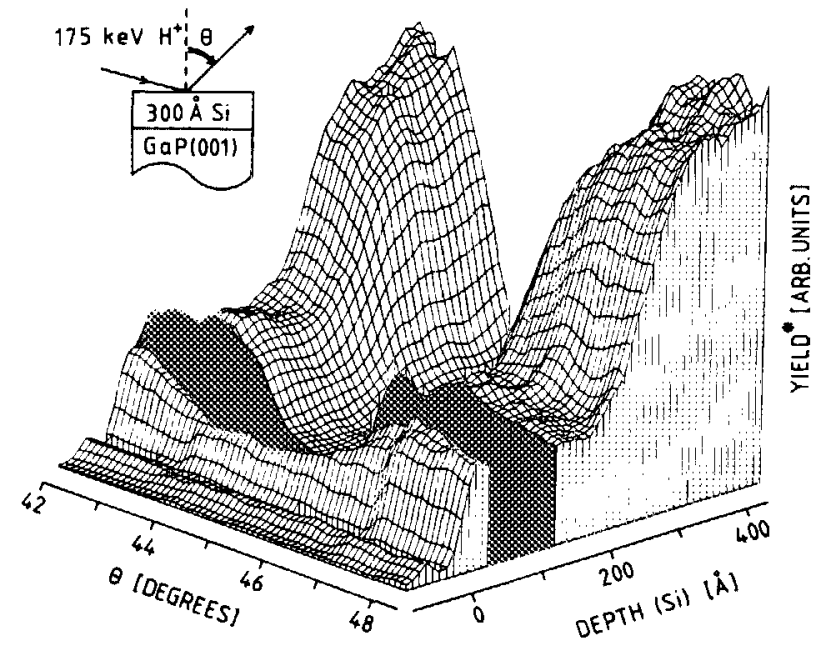

FIG. 7. Recorded multiangle backscatter spectrum with the electrostatic toroidal analyzer in the [011] position. The yield of backscattered protons, corrected for the angular dependent Rutherford cross section, is given as a function of the angle with the surface normal and of the depth in the Si layer. The depth window of $30-110 \AA$ is indicated by a shaded band.

In crystallographic directions a minimum in the backscattered yield is observed, because of blocking by the atomic rows. Our aim was to determine the exact angular position of the [011] blocking minimum. First, we aligned our detector with the [001] surface normal and measured the angular profile of the backscattered $\mathrm{H}^{+}$ions. Next we moved our detector, while keeping the crystal position fixed, exactly $45^{\circ}$ toward smaller scattering angles. This movement can be performed with an accuracy of $\pm 0.02^{\circ}$ by rotating a large $\operatorname{cog}$ wheel. In the new position again an angular profile was measured, now containing the [011] dip. The directions of the blocking minima in both profiles could be determined with an accuracy of $\pm 0.04^{\circ}$ with respect to the center of the detector. Use has been made of a fitting procedure with cubic splines and a varying number of points of support over an angular range of $4^{\circ}$. The observed difference in position of the minima shows the deviation of the angle between [001] and [011] directions from the value of $45^{\circ}$ it has in an unstrained crystal (Fig. 8). This is equal to the angular shift $\Delta \theta$ of the [011] direction, for the [001] surface normal direction is not affected by the lattice strain.

The irradiation dose during the measurement of one profile did not exceed $3 \times 10^{14} \mathrm{~cm}^{-2}$. It was verified that ion beam damage up to a dose of $10^{15} \mathrm{~cm}^{-2}$ had no measurable effect on the position of the blocking minima. The influence of the nonzero impact parameter and recoil of the $\mathrm{Si}$ atoms on the angular position of the minimum were calculated to be negliglible. Also, the effect of strain-induced bending of the substrate with film ${ }^{8}$ on $\Delta \theta$ is well below the experimental uncertainties. To detect possible bending of the substrate as a result of mounting, the measurements were repeated on different spots on the sample. No influence of this effect was observed on the orientation of the blocking dips. On one sample $(300 \AA)$ we have checked that angles between the $[001]$ surface normal and the [011] and [011] directions, respectively, are equal within the experimental error. 


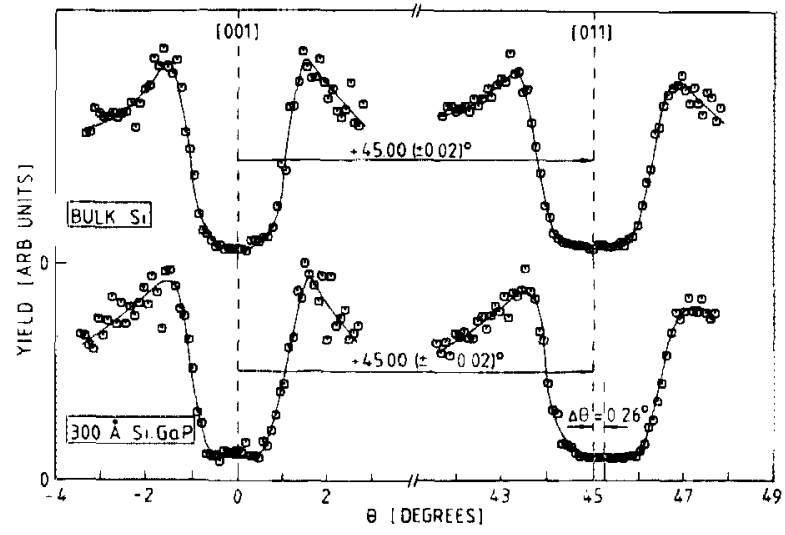

FIG. 8. Measured angular profiles of the [001] and [011] blocking minima in bulk Si (unstrained) and in a $300-\AA$ Si film on GaP(001). In the latter case the shift of the [011] axis is clearly visible.

The abovementioned procedure has the advantage that it is an absolute measurement of the strain in the film. Steering effects are a serious handicap of the method used by others ${ }^{23,24}$ in which substrate and film signals are obtained simultaneously by channeling angular scans, leading to systematically smaller values of $\Delta \theta$.

\section{DISCUSSION}

Our complementary measurements add up to a consistent picture of the strain and its relaxation with increasing thickness.

For the interpretation of the strain measurements we distinguish two regimes: a pseudomorphic regime $\left(t<t_{c} \approx 1000 \AA\right)$ and a regime in which part of the strain is relaxed by misfit dislocations $\left(t \geqslant t_{c}\right)$.

From the observed Raman line widths (Fig. 4, Table II) we conclude that the inhomogeneous strain in the layers increases slowly with increasing thickness. This is expected from the generation of gradually more misfit dislocations, as observed by TEM. The observations of Cerdeira et al. ${ }^{21}$ who report a reverse tendency, can be explained by the much larger lattice mismatch in their heterostructure. In that case, for a thin layer the interface is incommensurate and the disorder (localized near the interface) has reached its maximum, so that the relative amount of disorder decreases again for thicker layers. The same trend has been observed for the system $\mathrm{Si}: \mathrm{GaAs}(001){ }^{29}$

The measured frequency shift (Fig. 4, Table II) for the $500-\AA$ pseudomorphic layer (see Table I) is equal to $\delta \omega=2.6$ $( \pm 0.1) \mathrm{cm}^{-1}$, in good agreement with the value $\delta \omega=2.7$ $( \pm 0.5) \mathrm{cm}^{-1}$ calculated according to Eq. (15) taking $\epsilon_{\|}=f$ and $\alpha=0.77$. For thicker films the decrease of $\delta \omega$ shows the relaxation of the lattice strain (Fig. 9).

For pseudomorphic layers $\epsilon_{\|}$has its maximal value: $\epsilon_{\|}=f$. According to Eq. (16) with $\alpha=0.77$ as given by elasticity theory (Sec. IV), this would imply a maximum value of $\Delta \theta=0.19^{\circ}$. However, the measured values of $\Delta \theta$ are much larger $\left(0.26 \pm 0.04^{\circ}\right)$. This can only be explained by a higher value for $\alpha=1.5( \pm 0.5)$. The effects of doping (the Si layer contains $\approx 10^{18} \mathrm{~cm}^{-3}$ of $\mathrm{Ga}$ and about the same

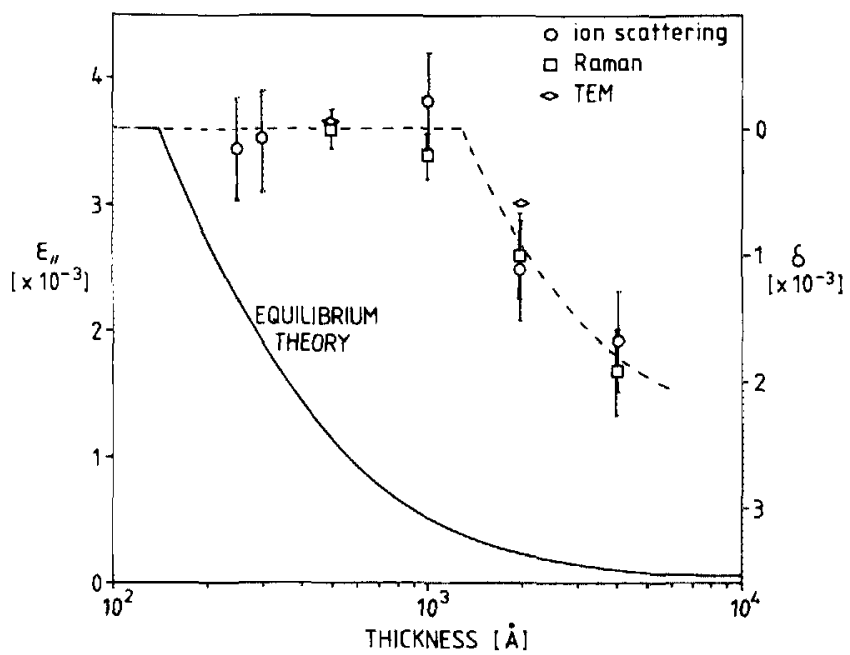

FIG. 9. Coherency strain $\left(\epsilon_{\|}\right)$vs Si layer thickness. Experimental results are compared with the values predicted by the equilibrium theory [Eq. (6)].

amount of $P$ atoms) and of stress on the elastic constants $\mathrm{s}^{30,31}$ are too small to explain this anomalously high $\alpha$ value.

It has to be pointed out that these blocking measurements are sensitive only to axial directions in the topmost part $(\approx 100 \AA)$ of the epilayer; they do not give an average direction over the film thickness. The reason for this is the steering effect, mentioned before, which is much stronger for medium energy protons than for high-energy $\mathrm{He}^{+}$ions. ${ }^{28}$ Although this effect did not distort the measured values in our experiment, it did limit the depth range over which the axial direction could be measured. We therefore, conclude that in the surface region of the strained films there is a larger contraction in the normal direction than expected from the Poisson effect. Therefore, there is a tendency for the outermost layers $(\approx 100 \AA)$ of the film to strive for volume- or bond-length conservation (see Sec. IV). The same behavior is observed in very thin $(\approx 10 \AA)$ silicide layers ${ }^{32,33}$ and apparently also in $100-\AA$ strained $\mathrm{Si}_{x} \mathrm{Ge}_{1-x}$ layers, ${ }^{11,12}$ though in the latter studies the effect is not explicitly mentioned.

If we nevertheless assume the perpendicular strain to be proportional to the parallel strain, we can also obtain quantitative information about the thickness dependence of $\epsilon_{\|}$. In Fig. 9 the parallel strain component $\epsilon_{\|}$is plotted as a function of layer thickness $t$. For the interpretation of the ion scattering results we used $\alpha=1.5$.

It is not possible to draw conclusions about the value of $\alpha$ from the Raman measurements. For larger values of $\alpha$ the error in the proportionality constant $\beta$ increases, e.g., for $\alpha=1.5, \beta$ becomes equal to $1.7( \pm 1.0)$. Note that apart from $\alpha$ the constants $p$ and $q$ in Eq. (15) also rely on a certain stress-strain relation.

The critical thickness $t_{c}$ at which relaxation of the tetragonal strain by the formation of misfit dislocations sets in is much larger than predicted by equilibrium theory (Sec. III). It is likely that this barrier, which is also observed in other heterostructures, ${ }^{8-12}$ originates from the mechanism of the dislocation formation. Viegers et al. ${ }^{34}$ proposed that misfit dislocations are generated by the nucleation of a $90^{\circ}$-Shockley partial dislocation followed by a $30^{\circ}$-Shockley partial, 
both on the same $\{111\}$ glide plane. Probably the barrier for the nucleation of these partial dislocations is too high to be overcome because of the low temperature of the $\mathrm{MBE}$ growth process, thus preventing thermodynamic equilibrium to be reached.

\section{CONCLUSIONS}

The lattice strain in thin Si films resulting from the small mismatch of the Si-GaP system has been determined using TEM, Raman spectroscopy, and Rutherford backscattering in conjunction with ion blocking.

The presence of the strain has an interesting side effect. Band-structure calculations have shown that for pseudomorphic Si films the degenerate energy levels of valence- and conduction-band at the center of the Brillouin zone are split and that the Si band gap is reduced by 60 to $80 \mathrm{meV}$. For tensile stress, as in the present case, this implies that intervalley scattering is strongly reduced and that only intravalley scattering determines the mobility. At room temperature both effects are of equal magnitude. ${ }^{35}$ As a consequence, a mobility enhancement by a factor of two is to be expected for pseudomorphic Si on $\mathrm{GaP}(001)$ as compared to bulk like Si. Future work on this aspect is important.

The results of the TEM and Raman spectroscopy measurements and the relative values of the ion-blocking results are consistent with each other and show that the relaxation of strain in the silicon films occurs by the generation of misfit dislocations at significantly larger thickness than predicted by equilibrium theory, suggesting the existence of a kinetical barrier.

The absolute values of the results of the ion-blocking experiments give a larger normal contraction of the topmost part of the strained layers than would follow from the Poisson effect. This indicates a tendency for the outermost layers ( $\approx 100 \AA$ ) of the film to strive for volume- and bond-length conservation.

\section{ACKNOWLEDGMENTS}

We would like to thank Dr. F. W. Saris for valuable discussions, L. J. H. Haenen for the Raman spectroscopy measurements, Dr. H. W. A. M. Rompa for the band-structure calculations and Dr. J. W. Bartels for the $\mathrm{x}$-ray diffraction results. This work is part of a joint research program of Philips and Fundamenteel Onderzoek der Materie (F.O.M.), with financial support from the Nederlandse organisatie voor Zuiver Wetenschappelijk Onderzoek (Z.W.O.).
'H. Kroemer, Proc. IEEE 70, 13 (1982).

${ }^{2}$ H. Schlotterer, Solid State Electron. 11, 947 (1968)

${ }^{3}$ G. C. Osbourn, R. M. Biefeld, and P. L. Gourley, Appl. Phys. Lett. 41, 172 (1982).

${ }^{4}$ G. C. Osbourn, J. Appl. Phys. 53, 1586 (1982).

${ }^{5}$ G. C. Osbourn, Phys. Rev. B 27, 5126 (1983).

${ }^{6}$ M. J. Ludowise, W. T. Dietze, C. R. Lewis, M. D. Camras, N. Holonyak, Jr., B. K. Fuller, and M. A. Nixon, Appl. Phys. Lett. 42, 487 (1983).

'J. H. van der Merwe and C. A. B. Ball, in Epitaxial Growth, edited by J. W. Matthews (Academic, New York, 1975), Part B, Chap. 6.

${ }^{8}$ E. Kasper, H. J. Herzog, and H. Kibble, Appl. Phys. 8, 199 (1975).

${ }^{9}$ E. Kasper and H.-J. Herzog, Thin Solid Films 44, 357 (1977).

${ }^{10} \mathrm{~J}$. C. Bean, T. T. Sheng, L. C. Feldman, A. T. Fiory, and R. T. Lynch, Appl. Phys. Lett. 44, 102 (1984).

${ }^{1}$ J. C. Bean, L. C. Feldman, A. T. Fiory, S. Nakahara, and I. K. Robinson, J. Vac. Sci. Technol. A 2, 436 (1984).

${ }^{12}$ A. T. Fiory, J. C. Bean, L. C. Feldman, and I. K. Robinson, J. Appl. Phys. 56, $1227(1984)$.

${ }^{13}$ T. de Jong, W. A. S. Douma, J. F. van der Veen, F. W. Saris, and J. Haisma, Appl. Phys. Lett. 42, 1037 (1983).

${ }^{14}$ T. de Jong, W. A. S. Douma, L. Smit, V. V. Korablev, and F. W. Saris, J. Vac. Sci. Technol. B 1, 888 (1983).

${ }^{15}$ G. A. Slack and S. F. Bartram, J. Appl. Phys. 46, 89 (1975).

${ }^{16} \mathrm{~J}$. H. van der Merwe, Surf. Sci. 31, 198 (1972).

${ }^{17}$ J. W. Matthews, in Dislocations in Solids, edited by F. R. N. Nabarro (North-Holland, Amsterdam, 1979) Vol. 2, Chap. 7.

${ }^{18}$ J. Hornstra and W. J. Bartels, J. Cryst. Growth 44, 513 (1978).

${ }^{19}$ T. R. Hart, R. L. Aggarwal, and B. Lax, Phys. Rev. B 1, 638 (1970).

${ }^{20}$ S. M. Sze, Physics of Semiconductor Devices (Wiley, New York, 1981).

${ }^{21}$ F. Cerdeira, A. Pinczuk, J. C. Bean, B. Batlogg, and B. A. Wilson, Appl. Phys. Lett. 45, 1138 (1984).

${ }^{22}$ E. Anastassakis, A. Pinczuk, E. Burstein, F. H. Pollak, and M. Cardona, Solid State Commun. 8, 133 (1970).

${ }^{23}$ W. K. Chu, C. K. Pan, and C.-A. Chang, Phys. Rev. B 28, 4033 (1983).

${ }^{24}$ S. T. Picraux, L. R. Dawson, G. C. Osbourn, R. M. Biefeld, and W. K. Chu, Appl. Phys. Lett. 43, 1020 (1983).

${ }^{25}$ F. W. Saris, Nucl. Instrum. Methods 194, 625 (1982).

${ }^{26}$ R. G. Smeenk, R. M. Tromp, H. H. Kersten, A. J. H. Boerboom, and F. W. Saris, Nucl. Instrum. Methods 195, 581 (1982).

${ }^{27}$ H. H. Andersen and J. F. Ziegler, Hydrogen Stopping Powers and Ranges in All Elements (Pergamon, New York, 1977).

${ }^{28}$ W. K. Chu, J. W. Mayer, and M. A. Nicolet, Backscattering Spectrometry (Academic, New York, 1978).

${ }^{29}$ P. C. Zalm, P. M. J. Marée and R. I. J. Olthof, Appl. Phys. Lett. 46, 597 (1985); C. W. T. Bulle-Lieuwma, P. C. Zalm, and M. P. A. Viegers, in Proceedings of the Fourth Oxford Conference on Microscopy of Semiconducting Materials, Oxford, March 1985 (to be published).

${ }^{30}$ N. S. Averkiev, Yu. V. Ilisavskiy, and V. M. Sternin, Solid State Commun. 52, 17 (1984).

${ }^{31}$ T. A. Fjeldly, F. Cerdeira, and M. Cardona, Solid State Commun. 12, 553 (1973).

${ }^{32}$ R. M. Tromp, E. J. van Loenen, R. G. Smeenk, F. W. Saris, F. Nava, and G. Ottaviani, Surf. Sci. 128, 224 (1983).

${ }^{33}$ E. J. van Loenen, A. E. M. J. Fischer, J. F. van der Veen, and F. Legoues, Surf. Sci. 154, 52 (1985).

${ }^{34}$ M. P. A. Viegers, C. W. T. Bulle-Lieuwma, P. C. Zalm, and P. M. J. Marée, in Proceedings of the Materials Research Society Symposia (NorthHolland, New York, 1985), Vol. 37, p.331.

${ }^{35}$ W. Zawadski, in Handbook of Semiconductors, edited by W. Paul (NorthHolland, Amsterdam, 1982), Vol. 1, p. 713. 